Andreas Vogt

\title{
Nichtschulbesuch als Kindeswohlgefährdung? Homeschooling in der Rechtsprechung des Bundesverfassungsgerichts und der Familiengerichte
}

\section{Zusammenfassung}

Ausgehend vom Status Quo der Schulpflicht in Deutschland gibt der Beitrag einen Überblick über Homeschooling in der Rechtsprechung des Bundesverfassungsgerichts und der Familiengerichte. Es zeigt sich, dass die Auffassung, Homeschooling bedeute unbesehen eine zu Eingriffen in die elterliche Sorge berechtigende Kindeswohlgefährdung, nicht in Einklang mit dem Elternrecht (Art. 6 Abs. 2 S. 1 GG) steht. Vielmehr entscheidend sind die Umstände des Einzelfalles, die das Familiengericht sorgfältig zu ermitteln hat.

Schlüsselwörter: Bundesverfassungsgericht, Elternrecht, elterliche Sorge, Familiengericht, Homeschooling, Kindeswohlgefährdung, Schulpflicht

\section{Not Going to School as a Child Welfare Risk?}

Homeschooling in the Case Law of the Federal Constitutional Court and the Family Courts

\begin{abstract}
Based on the status quo of compulsory school attendance in Germany, the article gives an overview of case law of the Federal Constitutional Court and the family courts on homeschooling. It turns out that the view that homeschooling implies a risk to the best interests of the child and justifies encroachments on parental rights is not in line with parental law (Art. 6, Par. 2, S. 1 of the Basic Law). Rather, the circumstances of the individual case, which the family court must carefully determine, are decisive.

Keywords: Federal Constitutional Court, parental rights, parental custody, family court, homeschooling, child endangerment, compulsory school attendance
\end{abstract}




\section{$1 \quad$ Einleitung}

Während fast alle europäischen und westlichen Staaten für eine Bildungs- oder Unterrichtspflicht optiert haben, besteht in den Ländern der Bundesrepublik Deutschland die Pflicht zum Besuch einer Schule. Minderjährige ab sechs Jahren müssen am Unterricht und an den sonstigen Veranstaltungen einer Schule regelmäßig teilnehmen. Die Erziehungsberechtigten sind sanktionsbewehrt ${ }^{1}$ verpflichtet, für die Erfüllung dieser Pflicht zu sorgen. Bis auf wenige Ausnahmen sehen die Schulgesetze unter besonderen Umständen die zwangsweise Zuführung vor (sog. Schulzwang). Den Schulbegriff definieren die Gesetze nicht ${ }^{2}$, sie gewähren nur die Freiheit der Wahl zwischen öffentlichen Schulen und staatlich genehmigten oder anerkannten Privatschulen (Ersatzschulen).

Die mithin rigide verstandene Schulpflicht bedeutet eine massenhafte und besonders intensive Verkürzung der Freiheit junger Menschen; in Spannung steht sie auch zu dem Erziehungsrecht der Eltern ${ }^{3}$. Nach herrschender Meinung sind diese Grund-

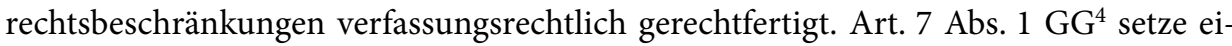
nen sog. Bildungs- und Erziehungsauftrag des Staates voraus, der durch die landesgesetzliche Schulpflicht konkretisiert werde; die mit ihr einhergehenden Eingriffe in das Selbstbestimmungsrecht des Kindes und das Elternrecht seien zur Erreichung des legitimen öffentlichen Zwecks geeignet, erforderlich und angemessen.

Nimmt ein schulpflichtiges Kind unentschuldigt nicht am Unterricht und den sonstigen Schulveranstaltungen teil, werden die Schulbehörde, die Ordnungsbehörde und/ oder das Jugendamt regelmäßig aktiv. Nicht selten wird ein familiengerichtliches Verfahren eröffnet. Das Familiengericht hat in Ausübung des staatlichen Wächteramts ${ }^{5}$ nach $₫ 1666$ Abs. 1 BGB bei Gefährdung des Kindeswohls die zur Abwendung der Gefahr erforderlichen Maßnahmen zu treffen, wenn die Eltern nicht willens oder in der Lage sind, die Gefahr abzuwenden. ${ }^{6}$ Was gilt, wenn das betroffene Kind aber zu Hause unterrichtet wird oder sonst schulbesuchsfrei lernt? Bedeutet Homeschooling

1 Erziehungsberechtigte, die ihre Pflicht vorsätzlich oder fahrlässig verletzen, begehen in allen Ländern der Bundesrepublik Deutschland eine Ordnungswidrigkeit, die mit einer Geldbuße geahndet werden kann. In Bremen, Hamburg, Hessen, Mecklenburg-Vorpommern und im Saarland ist das „Entziehen eines anderen (von) der Schulpflicht“ unter bestimmten Voraussetzungen sogar eine Straftat. Schulpflichtige, die zum Zeitpunkt der Tat bereits vierzehn Jahre alt sind, können in den meisten Ländern selbst mit Geldbuße belangt werden.

2 Zum Schulbegriff des Grundgesetzes jüngst Reimer (2021, S. 58 ff.).

3 Art. 6 Abs. 2 Satz 1 GG: „Pflege und Erziehung der Kinder sind das natürliche Recht der Eltern und die zuvörderst ihnen obliegende Pflicht."

4 „Das gesamte Schulwesen steht unter der Aufsicht des Staates.“

5 Art. 6 Abs. 2 Satz 2 GG: „Über ihre Betätigung wacht die staatliche Gemeinschaft.“

$6 \mathrm{Zu}$ den gerichtlichen Maßnahmen gehören insbesondere "Gebote, für die Einhaltung der Schulpflicht zu sorgen" und „die teilweise oder vollständige Entziehung der elterlichen Sorge“ (\$ 1666 Abs. 3 BGB). Maßnahmen, mit denen eine Trennung des Kindes von der elterlichen Familie verbunden ist, sind nur zulässig, wenn der Gefahr nicht auf andere Weise, auch nicht durch öffentliche Hilfen, begegnet werden kann ( $\$$ 1666a Abs. 1 Satz 1 BGB). 
stets eine Gefährdung des Kindeswohls? Während manche Familiengerichte dies bejaht haben, hat sich das Bundesverfassungsgericht dazu noch nicht geäußert.

\section{Rechtsprechung des Bundesverfassungsgerichts}

Die Erziehung des Kindes ist von Verfassungs wegen primär und bis zur Grenze der Kindeswohlgefährdung in die Verantwortung der Eltern gelegt, wobei dieses „natürliche Recht" den Eltern nicht vom Staate verliehen worden ist, sondern als vorgegebenes Recht anerkannt wird. ${ }^{7}$ Ein staatliches Erziehungsleitbild verfasst das Grundgesetz nicht. Eltern, deren sozioökonomische Verhältnisse, Werte und Verhaltensweisen sind grundsätzlich das Schicksal eines Kindes, d.h. Chance und Lebensrisiko zugleich. ${ }^{8}$ Zum Wächteramt des Staates gehört es darum nicht, die bestmögliche Förderung des Kindes und seiner Fähigkeiten sicherzustellen. ${ }^{9}$ Sorgen darf der Staat nur für die Einhaltung gewisser Mindeststandards elterlicher Verantwortung.

Mit diesen Direktiven stellt das Bundesverfassungsgericht hohe Anforderungen bereits an die Begründung einer Gefahrenlage im Sinne des $\$ 1666$ Abs. 1 BGB. Bislang nicht geäußert hat es sich zu der Frage, ob und inwieweit familiengerichtliche Maßnahmen verfassungsrechtlich gerechtfertigt sind, wenn Eltern nicht für den Schulbesuch sorgen, aber ihr Kind selbst zu Hause unterrichten oder es sonst schulbesuchsfrei lernen lassen. Alle in Fällen von Homeschooling ergangenen Judikate, die das Bundesverfassungsgericht überhaupt mit Gründen versehen hat ${ }^{10}$, betreffen nicht familiengerichtliche Beschlüsse, sondern Maßnahmen der Schul- und Ordnungsbehörden sowie Entscheidungen der Verwaltungsgerichte und der Fachgerichte in Bußgeldund Strafsachen.

\subsection{Fall Bartmann (1986)}

Im Fall Bartmann beriefen sich die Beschwerdeführer ${ }^{11}$, gegen die im Ausgangsverfahren Geldbußen verhängt worden waren, auf den Respekt vor dem Willen ihres sechs Jahre alten Sohnes, nicht zur Schule zu gehen. Die 2. Kammer des Ersten Senats des Bundesverfassungsgerichts (Beschluss vom 05.09.1986 - 1 BvR 794/86) nahm die Verfassungsbeschwerde nicht zur Entscheidung an. „Die allgemeine Schulpflicht und die sich daraus ergebenden weiteren Pflichten beschränken in zulässiger Weise das in

7 BVerfGE 59, 360. Den überpositiven Charakter des Elternrechts hat das Bundesverfassungsgericht bereits in BVerfGE 1, 14 kräftig herausgestrichen.

8 BVerfG, FamRZ, 2010, 713; 2017, 1577.

9 BVerfG, FamRZ, 2014, 907; 2015, 112.

10 Die Verfassungsbeschwerde bedarf der Annahme zur Entscheidung ( $\$ 93$ a Abs. 1 BVerfGG i.V.m. Art. 94 Abs. 2 GG). Die Ablehnung der Annahme muss nicht begründet werden ( $\$$ 93d Abs. 1 Satz 3 BVerfGG).

11 Anmerkung der Redaktion: Auf Wunsch des Autors wird ausschließlich das generische Maskulinum verwendet. 
Art. 6 Abs. 2 Satz 1 GG gewährleistete elterliche Bestimmungsrecht über die Erziehung des Kindes.“ Die Beschwerdeführer, die nur „ihre eigenen pädagogischen Vorstellungen durchsetzen“ wollten, verkennten die rechtliche und tatsächliche Ausgangslage. Von einer freien Entscheidung gegen den Schulbesuch, die es zu respektieren gelte, könne bei einem Sechsjährigen keine Rede sein; das Grundgesetz selbst setze die „Notwendigkeit einer Bevormundung von Kindern“ voraus.

\subsection{Fall Treu (1989)}

Derselbe Spruchkörper (2. Kammer des Ersten Senats) führte im Nichtannahmebeschluss vom 21.04.1989 - 1 BvR 235/89 (Fall Treu) aus, allein das pauschale Vorbringen der Beschwerdeführer, die Schule mache ihren Sohn krank und das derzeitige Schulsystem erwecke Ängste in den Schülerinnen und Schülern, biete „keinen konkreten Anhaltspunkt für eine verfassungsrechtliche Kontrolle“. Eltern hätten keinen Anspruch darauf, dass Schule so organisiert und der Unterricht so gestaltet sein muss, wie es ihren eigenen pädagogischen Anschauungen entspricht. Darüber hinaus hätten Eltern es „hinzunehmen, dass der Staat seinen verfassungsrechtlichen Erziehungsauftrag nach seinen bildungspolitischen Vorstellungen zu verwirklichen sucht“. Ein gegen den Schulbesuch gerichtetes Selbstbestimmungsrecht von Kindern sei mit dem Grundgesetz nicht vereinbar. Auf die Freiheit ihres Gewissens könnten sich die sorgeverpflichteten Beschwerdeführer nicht berufen.

\subsection{Fall Konrad (2003)}

Im Fall Konrad richteten sich die Beschwerdeführer gegen die Ablehnung einer Genehmigung zur Erteilung von elterlichem Heimunterricht außerhalb staatlicher oder privater Schulen. Die 2. Kammer des Ersten Senats des Bundesverfassungsgerichts nahm auch diese Verfassungsbeschwerde nicht zur Entscheidung an (Beschluss vom 29.4.2003 - 1 BvR 436/03) und führte zur Begründung aus:

„Die Pflicht zum Besuch der staatlichen Grundschule dient dem legitimen Zweck der Durchsetzung des staatlichen Erziehungsauftrags und ist zur Erreichung dieses Ziels geeignet und erforderlich. Dieser Auftrag richtet sich nicht nur auf die Vermittlung von Wissen, sondern auch auf die Heranbildung verantwortlicher Staatsbürger, die gleichberechtigt und dem Ganzen gegenüber verantwortungsbewusst an den demokratischen Prozessen in einer pluralistischen Gesellschaft sollen teilhaben können. [...] soziale Kompetenz im Umgang auch mit Andersdenkenden, gelebte Toleranz, Durchsetzungsvermögen und Selbstbehauptung einer von der Mehrheit abweichenden Überzeugung können effektiver eingeübt werden, wenn Kontakte mit der Gesellschaft und den in ihnen vertretenen unterschiedlichsten Auffassungen nicht nur gelegentlich stattfinden, sondern Teil einer mit dem regelmäßigen Schulbesuch verbundenen Alltagserfahrung sind.“ 


\subsection{Fall Bauer (2006)}

Die Verfassungsbeschwerde im Fall Bauer wurde von Eltern erhoben, die wegen Entziehung ihrer Kinder (von) der Schulpflicht gemäß $₫ 182$ Abs. 1 HSchG (Hessisches Schulgesetz) ${ }^{12}$ schuldig gesprochen und (lediglich) mit Strafvorbehalt verwarnt worden waren. Im Nichtannahmebeschluss vom 31.05.2006 - 2 BvR 1693/04 - übernahm die 1. Kammer des Zweiten Senats des Bundesverfassungsgerichts die oben zitierte Konrad-Formel und führte weiter aus, dass der Widerspruch zwischen dem strafbewehrten Handlungsgebot - die Teilnahme ihrer Kinder am Unterricht einer anerkannten Schule - und den eigenen Glaubensüberzeugungen sich im Falle der Beschwerdeführer nicht als derart ausweglos erweise, dass diese berechtigt gewesen wären, dem Verbotsgesetz zuwider zu handeln.

\subsection{Fall Schaum (2014)}

Ebenfalls die Strafbarkeit von Eltern betraf die Verfassungsbeschwerde im Fall Schaum. Obwohl die Ausgangsgerichte, die die Beschwerdeführer gemäß $₫ 182$ Abs. 1 HSchG schuldig gesprochen und zu Geldstrafen verurteilt hatten, gerade nicht festgestellt hatten, dass das Wohl der Kinder durch die häusliche Unterrichtung gefährdet war, nahm die 2. Kammer des Zweiten Senats des Bundesverfassungsgerichts auch diesen außerordentlichen Rechtsbehelf nicht zur Entscheidung an. Nach Ansicht der Kammer, die in dem Beschluss vom 15.10.2014 - 2 BvR 920/14 auch breite Ausführungen zur Gesetzgebungsbefugnis des Landes macht und die Strafnorm des $\$ 182$ Abs. 1 HSchG scharf abgrenzt gegenüber $\$ 171$ StGB (Verletzung der Fürsorge- und Erziehungspflicht), verhindert

„selbst ein mit erfolgreichen Ergebnissen einhergehender Hausunterricht [...] nicht, dass sich die Kinder vor einem Dialog mit Andersdenkenden und -gläubigen verschließen, und ist deshalb nicht geeignet, die insbesondere in einer Klassengemeinschaft gelebte Toleranz gegenüber einem breiten Meinungsspektrum nachhaltig zu fördern“.

\section{Familiengerichtliche Rechtsprechung}

Die jüngere Judikatur in Familiensachen ist uneinheitlich. ${ }^{13}$ Während einige Fachgerichte die früher anerkannte Gleichung Nichtschulbesuch $=$ Kindeswohlgefährdung aufgegeben haben, verteidigen andere sie weiterhin. In die zuletzt genannte Kategorie

12 „Wer einen anderen der Schulpflicht dauernd oder hartnäckig wiederholt entzieht, wird mit Freiheitsstrafe bis zu sechs Monaten oder mit Geldstrafe bis zu einhundertachtzig Tagessätzen bestraft."

13 Zur familiengerichtlichen Rechtsprechung bis 2015 vgl. Vogt (2016, S. 95 ff.). 
fallende Sorgerechtsentscheidungen referieren gewöhnlich zwei Beschlüsse des Bundesgerichtshofs vom 11.07.2007 (XII ZB 41/07 und XII ZB 42/07) ${ }^{14}$, allerdings regulieren sie ihr Zwischenergebnis (Nichtschulbesuch = Kindeswohlgefährdung) am Grundsatz der Verhältnismäßigkeit und gelangen so häufig zu dem Ergebnis, von familiengerichtlichen Maßnahmen abzusehen.

\subsection{Oberlandesgericht Nürnberg (Beschluss vom 15.09.2015 - 9 UF 542/15 - und Beschluss vom 18.11.2016 - 9 UF 551/16)}

In einem turbulenten Fall aus Franken wurde das Kindesschutzverfahren 2012 eingeleitet, weil das betroffene im Frühjahr 2006 geborene Kind nicht am Schulunterricht teilnahm. Im März 2015 entzog das Amtsgericht - Familiengericht - gemäß $\$ 1666$ Abs. 1 BGB der allein sorgeberechtigten Kindesmutter Teile der elterlichen Sorge („Recht zur Regelung schulischer Angelegenheiten“, „Aufenthaltsbestimmungsrecht, soweit es die Durchführung des Schulbesuchs betrifft“, „Recht Hilfe zur Erziehung zu beantragen"), ordnete insoweit Ergänzungspflegschaft an und ermächtigte den Ergänzungspfleger,

„die Herausgabe des Kindes zum Schulbesuch notfalls unter Einsatz von Gewalt und mittels Betreten und Durchsuchung der Wohnungen beider Eltern sowie unter Inanspruchnahme der Hilfe des Gerichtsvollziehers oder der Polizei zu erzwingen".

Die dagegen eingelegten Beschwerden der Kindeseltern und des weitere Sorgerechtseingriffe begehrenden Jugendamts wies das Oberlandesgericht Nürnberg - 9. Zivilsenat und Senat für Familiensachen - durch Beschluss vom 15.09.2015 - 9 UF 542/15 als unbegründet zurück.

Nachdem das betroffene Kind am Schulunterricht lediglich von April 2015 bis Oktober 2015 teilgenommen hatte, hob das Amtsgericht im März 2016 auf Antrag der Kindesmutter seinen rechtskräftig gewordenen Beschluss vom Vorjahr auf und sah von familiengerichtlichen Maßnahmen ab. Dagegen legte das Jugendamt Beschwerde ein; zur Begründung trug es insbesondere vor, der bisherige Entzug der Teilsor-

14 Danach kann ein „Missbrauch der elterlichen Sorge, der das Wohl der Kinder nachhaltig gefährdet" und Maßnahmen des Familiengerichts nach $\$ \$ 1666,1666$ a BGB erfordert, darin liegen, dass sich Eltern beharrlich weigern, ihre Kinder der öffentlichen Grundschule oder einer anerkannten Ersatzschule zuzuführen. Von der Einholung eines Sachverständigengutachtens, meint der Bundesgerichtshof (a. a. O.) weiter, habe das Beschwerdegericht im Ausgangsverfahren absehen dürfen. „Der Erhebung eines solchen Sachverständigengutachtens bedurfte es [...] nicht, da sich die vom Oberlandesgericht - in Anlehnung an die Rechtsprechung des Bundesverfassungsgerichts - geschilderten Vorzüge eines nicht hausgebundenen Unterrichts ebenso wie die relativen Nachteile eines Hausunterrichts dem tatrichterlichen Sachverstand ohne weiteres erschließen und sich zudem mit der Einschätzung des deutschen Schulgesetzgebers wie auch des Bundesverfassungsgerichts decken." 
gerechtsbereiche sei unzureichend, es bedürfe eines umfassenderen Rechts des Ergänzungspflegers auf Unterbringung notfalls in einer Jugendhilfeeinrichtung, um den Schulbesuch des Betroffenen sicherzustellen. Diesem Rechtsmittel aber verhalf das Oberlandesgericht Nürnberg - 9. Zivilsenat und Senat für Familiensachen - nicht zum Erfolg (Beschluss vom 18.11.2016 - 9 UF 551/16).

Nach Auffassung des Senats bedeutet der Nichtbesuch einer Schule eine Kindeswohlgefährdung. Zwar sei in dem Fall kein Zurückstehen hinter dem Wissensstand und den kognitiven Fähigkeiten Gleichaltriger festzustellen. Der Betroffene, an dessen sozialer Kompetenz nach den amtsgerichtlichen Feststellungen keine Zweifel bestünden, „wirkt in seiner geistigen Entwicklung durchaus altersgerecht“. Darauf komme es jedoch nicht an. $\mathrm{Ob}$ und mit welchem Erfolg es den Eltern bisher gelungen ist, einen altersangemessenen Lern- und Bildungsstand ihres Kindes zu gewährleisten, sei nicht entscheidend. Denn es gelte „der staatliche Bildungs- und Erziehungsauftrag der Schule" (Art. 7 Abs. 1 GG), und dieser richte sich über die Vermittlung von Wissen hinaus auch auf die „Aufgabe, den Kindern durch einen gemeinsamen Schulbesuch die Gelegenheit zu geben, in das Gemeinschaftsleben in der Gesellschaft hineinzuwachsen“.

Gleichwohl war nach Überzeugung des Senats ein Eingriff in das elterliche Sorgerecht „nicht mehr aufrechtzuerhalten“. Erfolgreich abgewendet werden könne die Kindeswohlgefährdung nur durch eine Trennung des Kindes von seinen Eltern, aber eine solche Maßnahme zur Durchsetzung der Schulpflicht „erscheint [...] nicht mehr verhältnismäßig“;

„Eine Herausnahme aus diesem stabilen Umfeld verbunden mit einer dauerhaften Fremd- oder Internatsunterbringung würde [...] zu einer eigenständigen erheblichen Beeinträchtigung des Kindeswohls führen, die den hierdurch erstrebten Zweck, nämlich den Schulbesuch des Kindes sicherzustellen und die aus der Nichtbeschulung resultierenden Nachteile abzuwenden, im Ergebnis nicht rechtfertigen kann."

Ein geringerer Eingriff in das elterliche Sorgerecht sei ebenfalls „nicht mehr gerechtfertigt“, nachdem sich der vom Amtsgericht bereits im März 2015 beschlossene teilweise Sorgerechtsentzug ,als ungeeignet zur Abwendung der bestehenden Kindswohlgefahr erwiesen“ habe: „Die schulverweigernde Haltung der Eltern hat sich hierdurch nicht verändert.“ Wegen der „weltanschaulich begründeten beharrlichen Weigerung der Eltern, den Schulbesuch ihres Kindes zu gewährleisten", stelle sich auch das familiengerichtliche Gebot, für die Einhaltung der Schulpflicht zu sorgen (\$ 1666 Abs. 3 Ziffer 2 BGB) als „nicht zielführend“ dar. 


\subsection{Oberlandesgericht Schleswig (Beschluss vom 27.12.2018 - 10 UF 176/18)}

Auch nach Auffassung des schleswig-holsteinischen Oberlandesgerichts - 2. Familiensenat - stellt der unterbliebene Schulbesuch eines Kindes ab der 6. Klasse eine gegenwärtige und erhebliche Kindeswohlgefährdung dar. Der „staatliche Bildungs- und Erziehungsauftrag der Schule" diene über die Vermittlung von Wissen hinaus auch der Aufgabe der Integration „in ein Gemeinschaftsleben außerhalb der Familie“. Familiengerichtlichen Maßnahmen zur Durchsetzung der Schulpflicht könne aber der Grundsatz der Verhältnismäßigkeit entgegenstehen. Dies könne der Fall sein, wenn eine 14-Jährige wiederholt und nachhaltig äußert, nur noch „selbstbestimmt“ außerhalb der Schule lernen zu wollen, sie die sog. „Kulturtechniken“ beherrscht und über angemessenes Grundwissen und angemessene Sozialkompetenz verfügt. ${ }^{15}$ Der wiederholt und nachhaltig geäußerte Wille der bei Erlass der obergerichtlichen Entscheidung bereits 15 Jahre alten Betroffenen, bis auf weiteres nur noch „selbstbestimmt“ außerhalb der Schule zu lernen, könne familienrechtlich nicht unbeachtet bleiben. Der Kindeswille sei Ausdruck der verfassungsrechtlich zu achtenden Selbstbestimmung des Kindes und erlange mit dem Alter des Kindes zunehmend an Bedeutung. Ein gegen den ernsthaften Widerstand eines Kindes erzwungenes Verhalten könne durch die Erfahrung der Missachtung der eigenen Persönlichkeit unter Umständen mehr Schaden verursachen als Nutzen bringen.

\subsection{Oberlandesgericht Düsseldorf (Beschluss vom 25.07.2018 - 2 UF 18/17), Oberlandesgericht Naumburg (Beschluss vom 02.05.2019 - 3 UF 142/18) und Oberlandesgericht Hamm (Beschluss vom 11.10.2019 - 3 UF 116/19)}

Verzichtet auf die Rezeption der Gleichung Nichtschulbesuch = Kindeswohlgefährdung hat der 2. Senat für Familiensachen des Oberlandesgerichts Düsseldorf. In diesem Fall hatte Ende 2016 das Amtsgericht - Familiengericht - der allein sorgeberechtigten Kindesmutter unter Fristsetzung insbesondere aufgegeben, das betroffene 2005 geborene Kind an einer öffentlichen Schule oder einer anerkannten Ersatzschule anzumelden und es einer Beschulung zuzuführen. Im Verfahren über die dagegen eingelegten Beschwerden des Kindes und seiner Mutter erachtete der angerufene Senat nach Einholung von Sachverständigengutachten und mündlicher Verhandlung die Rechtsmittel für begründet. In den Gründen des obergerichtlichen Beschlusses vom 25.07.2018 - 2 UF 18/17 - wird ausgeführt: Dass der unterdessen 13 Jahre alte Betroffene

15 Vgl. FamRZ 2019, 453. 
„bislang weder eine öffentliche Schule noch eine staatlich anerkannte Ersatzschule besucht hat und anzunehmen ist, dass er dies auch in Zukunft nicht tun wird, reicht allein für familiengerichtliche Maßnahmen nach $₫ 1666$ BGB nicht aus".

Der Entwicklungsstand des Kindes „in körperlicher, kognitiver, sprachlicher, motivationaler, emotionaler und sozialer Hinsicht [sei] als normgerecht einzustufen und in jeder Hinsicht altersgemäß“. Auch vor dem Hintergrund des Ergebnisses des schulpsychologischen Gutachtens lasse sich „bei weiterhin unterbleibendem Schulbesuch“ eine „erhebliche Schädigung [...] nicht mit der erforderlichen Sicherheit feststellen“.

Dieser Entscheidung haben sich das Oberlandesgericht Naumburg - 1. Senat für Familiensachen, mit Beschluss vom 02.05.2019 - 3 UF 142/18 - und das Oberlandesgericht Hamm - 3. Familiensenat, mit Beschluss vom 11.10.2019 - 3 UF 116/19 - angeschlossen. Nach den Leitsätzen der zuletzt genannten Entscheidung ${ }^{16}$ stellt allein die Weigerung der Eltern, ihr Kind auf einer öffentlichen Schule oder einer staatlich anerkannten Ersatzschule beschulen zu lassen, keine Kindeswohlgefährdung dar, die sorgerechtliche Maßnahmen nach $₫ 1666$ BGB rechtfertigen würde, wenn sie dem Kind durch Heimbeschulung hinreichend Wissen vermitteln und keine konkreten Anhaltspunkte für eine Gefährdung der körperlichen, kognitiven, sprachlichen, motivationalen, emotionalen und sozialen Entwicklung des Kindes vorliegen. Zur Beurteilung möglicher, sich aus der fehlenden Regelbeschulung ergebender kindeswohlgefährdender Umstände bedürfe es einer sachverständigen Begutachtung, die auch die Ermittlung des bisherigen Wissensstandes umfasst.

\section{Ergebnis}

Die Auffassung, Homeschooling bedeute per se eine Kindeswohlgefährdung, findet in der Rechtsprechung des Bundesverfassungsgerichts keine Stütze. Sie wird in der familiengerichtlichen Judikatur nur noch selten vertreten, jüngst vom Oberlandesgericht Celle (Beschluss vom 02.06.2021 - 21 UF 205/20), und ist zu Recht im Rückzug begriffen. Für die Durchsetzung der Pflicht zum Besuch einer Schule im organisatorisch-formalen Sinne mag es sozialpolitische Argumente geben. Solche überindividuellen Interessen müssen im Kontext der tatbestandlich begrenzten Ermächtigung der Familiengerichte aber dahingestellt bleiben. Aspekte des Gemeinwohls dürfen wegen des Telos des $\$ 1666$ BGB, der Ideologieanfälligkeit des unbestimmten Kindeswohlbegriffs und der als Abwehrrechte gegen den Staat zu begreifenden Grundrechte nicht dazu führen, dass auf die fundierte Prüfung verzichtet wird, ob die Nichterfüllung der Schulpflicht im individuellen Fall das Wohl des betroffenen Kindes gegenwärtig und nachhaltig gefährdet (vgl. Fröschle, 2018, S. 219). Der Schluss vom Homeschoo-

16 Vgl. FamRZ, 2020, 344. 
ling auf eine Kindeswohlgefährdung im Sinne des $₫ 1666$ Abs. 1 BGB ist keineswegs zwingend; vielmehr entscheidend sind die Umstände des Einzelfalls, die das Familiengericht sorgfältig zu ermitteln hat, etwa durch Einholung eines Sachverständigengutachtens. Damit nicht beantwortet sind rechtsdogmatische Fragen, die sich jenseits des Familienrechts insbesondere im Schulrecht stellen, und die rechtspolitische Frage, ob die Schulpflicht weniger eng begriffen und durch eine Bildungspflicht ersetzt werden sollte (vgl. Reimer in diesem Heft, S. 409-421).

\section{Literatur und Internetquellen}

Hinweis der Redaktion: Als Hilfestellung sind die wesentlichen rechtlichen Regelungen, auf die in den Beiträgen des Heftes Bezug genommen wird, in einem das Heft begleitenden Online-Anhang abgedruckt. Er ist unter folgendem Link abrufbar: https://www.waxmann. com/artikelART104608

Fröschle, T. (2018). Sorge und Umgang in der Rechtspraxis (2., neu bearb. Aufl.). Bielefeld: Gieseking.

Reimer, F. (2021). Der verfassungsrechtliche Schulbegriff: neuer Wein in neue Schläuche!, Recht der Jugend und des Bildungswesens, 69 (1), 58-77.

Vogt, A. (2016). Die Schulpflicht vor Gericht. Freilernen und Homeschooling in der Rechtsprechung. In M. Kern (Hrsg.), Selbstbestimmte und selbstorganisierte Bildung versus Schulpflicht. Betrachtungen zum Spannungsverhältnis zwischen Schulbesuchspflicht und den Grundrechten der jungen Menschen. Beiträge zur Frage des Rechts, frei sich zu bilden - Band 1 (Tagungsband zum Kolloquium vom 18. Juli 2014 in Gießen) (S. 85104). Leipzig: Tologo.

Andreas Vogt, Dr. iur., geboren 1973, Rechtsanwalt

E-Mail: kanzlei@vogt-recht.de

Korrespondenzanschrift: Niederhoner Str. 20, 37269 Eschwege 